\title{
Exosomes Derived from Human Umbilical Cord Mesenchymal Stem Cells Regulate Macrophage Polarization to Attenuate Systemic Lupus Erythematosus-Associated Diffuse Alveolar Hemorrhage in Mice
}

\author{
Xun Chen*, Qing Wei*, Hongmei Sun, Xiaobo Zhang, Changrong Yang, Ying Tao, Guangmin Nong \\ Department of Pediatrics, The First Affiliated Hospital of Guangxi Medical University, Guangxi, China
}

\begin{abstract}
Background and Objectives: To investigate the effect and the underlying mechanism of exosomes secreted by human umbilical cord mesenchymal stem cells (hUCMSCs) on diffuse alveolar hemorrhage (DAH) in murine lupus.

Methods and Results: Exosomes were extracted from cultured hUCMSCs by ultracentrifugation. The expressions of exosome markers (Alix, CD63 and TSG101) were measured for identification of hUCMSC-derived exosomes (hUCMSC-exosomes). The alveolar hemorrhage of DAH mice was revealed by H\&E staining. The primary alveolar macrophages were isolated from bronchoalveolar lavage fluid (BALF) of DAH mice. The expressions of M1 macrophage markers (iNOS, IL-6, TNF- $\alpha$ and IL-1 $\beta$ ) and M2 macrophage markers (Arg1, IL-10, TGF- $\beta$ and chi313) were detected. Flow cytometry measured the ratio of M1/M2 macrophages. ELISA measured the secretion of pro-inflammatory cytokines (IL-6 and TNF- $\alpha$ ) and anti-inflammatory cytokines (IL-10 and TGF- $\beta$ ). DAH mice had hemorrhage and small-vessel vasculitis in the lung, with neutrophil and monocyte infiltration observed around the capillary and small artery. Furthermore, increases of IL- 6 and TNF- $\alpha$, and decreases of IL-10 and TGF- $\beta$ were detected in the BALF of DAH mice. M1 makers were overexpressed in alveolar macrophages of DAH mice while M2 makers were lowly expressed. DAH mice had a higher proportion of $\mathrm{M} 1$ macrophages than M2 macrophages. After hUCMSC-exosome or methylprednisolone treatment in DAH mice, the alveolar injuries and inflammatory responses were attenuated, and the proportion of M2 macrophages was increased.

Conclusions: hUCMSC-exosomes attenuate DAH-induced inflammatory responses and alveolar hemorrhage by regulating macrophage polarization.
\end{abstract}

Keywords: Human umbilical cord mesenchymal stem cells, Exosome, Systemic lupus erythematosus, Diffuse alveolar hemorrhage, M1 macrophage, M2 macrophage

Received: September 28, 2020, Revised: December 24, 2020, Accepted: January 30, 2021, Published online: April 30, 2021 Correspondence to Guangmin Nong

Department of Pediatrics, The First Affiliated Hospital of Guangxi Medical University, No. 6, Shuanyong Road, Nanning, Guangxi 530021, China

Tel: +86-13878807055, Fax: +86-13878807055

E-mail: ngm8525@163.com

${ }^{*}$ These authors contributed equally to this work.

(a) This is an open-access article distributed under the terms of the Creative Commons Attribution Non-Commercial License (http://creativecommons.org/ licenses/by-nc/4.0/), which permits unrestricted non-commercial use, distribution, and reproduction in any medium, provided the original work is properly cited.

Copyright (c) 2021 by the Korean Society for Stem Cell Research

\section{Introduction}

Diffuse alveolar hemorrhage (DAH) is an uncommon but fatal complication of systemic lupus erythematosus (SLE) (1). The exact pathogenesis of DAH remains obscure but it is generally believed that immune complexes-induced pulmonary capillaritis or bland hemorrhage is responsible (2). The sudden outbreak and rapid progression of SLE-associated DAH (SLE-DAH) as well as its non-specific clinical symptoms impede the early diagnosis, and consequently lead to dismal patient survival 
(3). Despite the rare incidence of DAH in SLE, the mortality rate of SLE-DAH can reach up to $90 \%$ (4). Therefore, novel therapeutic strategy for SLE-DAH is still needed to improve the clinical outcomes of DAH treatment.

Stem cell therapy has gained popularity in a variety of diseases and injuries such as Parkinson's disease, type 1 diabetes and spinal cord injury (5-7). Mesenchymal stem cells (MSCs) are the predominant sources for cell-based therapy in various clinical conditions due to their capacity of differentiation and self-renewal (8). Among the MSC populations, human umbilical cord mesenchymal stem cells (hUCMSCs) are extracted from donor sources through non-invasive procedures and exert immunosuppressive and hematopoiesis-supportive effects (9). With the immunomodulatory potential, hUCMSCs have been increasingly investigated in the context of immunologic diseases including SLE (10). hUCMSCs have been reported to improve the clinical condition of patients with SLE-DAH (11). However, the specific mechanism underlying the effect of hUCMSCs on SLE-DAH remains largely unknown.

Exosomes, belonging to the smallest members of the extracellular vesicle (EV) family, are released to the extracellular environment after fusion of multivesicular bodies with the plasma membrane (12). These membrane vesicles are implicated in a wide range of biological processes $(13,14)$. hUCMSCs-derived exosomes (hUCMSC-exosomes), for instance, were shown to exert biological functions in different diseases, such as kidney disease, ocular disease, Alzheimer's disease and inflammatory bowel disease (15-19). Additionally, hUCMSC-exosomes can suppress inflammatory response in the lesion area to ameliorate spinal cord injury (20). Also, hUCMSC-exosomes are enriched with microRNAs that suppress myofibroblast differentiation during wound repair (21). Importantly, MSCderived EVs have shown great potential as therapeutic agents for autoimmune and inflammatory diseases such as SLE (22). Although the therapeutic effect of hUCMSCexosomes has not been discussed in the context of SLEDAH yet, the anti-inflammatory potential of hUCMSCexosomes has been proved in many other diseases. Therefore, it is reasonable to consider hUCMSC-exosomes may act on DAH by attenuating inflammatory responses.

Macrophages are originated from monocytes which are distributed in different tissues of the body and regulate many organ systems such as innate and adaptive immunity (23). Macrophage includes two main phenotypes: inflammatory M1 and anti-inflammatory M2 (24). Macrophages undergo phenotypic polarization to obtain the required functional phenotype in response to various environmental stimuli (25). Macrophage polarization plays a critical role in development of inflammatory and neoplastic diseases (26). Recent studies indicate that MSC-exosomes can suppress inflammation by regulating macrophage polarization in many injuries, such as myocardial infarction (27), myocardial ischaemia/reperfusion injury (28) and colitis (29). Although the pathogenesis of DAH is not fully disclosed, Zhuang et al. (30) claimed that DAH was macrophagedependent. The present study aims at uncovering whether hUCMSC-exosomes exert function by regulating macrophage polarization in SLE-DAH.

\section{Materials and Methods}

\section{Laboratory animals}

Forty heathy female C57BL/6J mice (specific pathogen free), aged $8 \sim 12$ weeks with an average weight of $20 \sim 22$ g, were purchased from Hunan SJA Laboratory Animal Co., Ltd. (Hunan, China) and fed in the Laboratory Animal Center of Guangxi Medical University. All mice were raised in same environment at $22 \sim 26^{\circ} \mathrm{C}$ with adequate lighting. The mice were provided with enough food and water, and the padding was replaced at regular intervals to keep clean and dry. The design of this research was approved by the Animal Ethics Committee of the First Affiliated Hospital of Guangxi Medical University.

\section{Cell culture}

hUCMSCs were purchased from Saliai Stem Cell Science and Technology Co., Ltd. (Guangzhou, China) and cultured in DMEM/F12 (gibco, USA) consisting of $10 \%$ fetal calf serum (FBS) and $1 \%$ penicillin-streptomycin. The cells were placed into an incubator which was maintained at $37^{\circ} \mathrm{C}$ with $5 \% \mathrm{CO}_{2}$ and $95 \%$ humidity. Cells at the logarithmic phase were collected for experiments.

\section{Differential ultracentrifugation for extraction of exosomes}

After three to six passages, well-conditioned hUCMSCs were selected for exosome extraction. Medium of the cells was replaced with DMEM/F12 without serum or penicillin/streptomycin when the cells reached $80 \%$ confluence. About $48 \mathrm{~h}$ later, $750 \mathrm{ml}$ of culture supernatant was collected and centrifuged at $4^{\circ} \mathrm{C}, 300 \mathrm{~g}$ for $10 \mathrm{~min}$. Supernatant obtained was then centrifuged at 2,000 $\mathrm{g}$ for $10 \mathrm{~min}$. Supernatant obtained from the former step was centrifuged at 10,000 g for $30 \mathrm{~min}$. At last, the supernatant was centrifuged at $140,000 \mathrm{~g}$ for $90 \mathrm{~min}$. An ultra-centrifuge (optima XE-100, Beckman, USA) was used here for exosome isolation. Sediments were obtained after the final 
centrifugation, from which exosomes were obtained. The sediments were washed in PBS buffer and resuspended. The suspension was centrifuged at $140,000 \mathrm{~g}$ for $90 \mathrm{~min}$ and the sediments were resuspended in $100 \mu 1$ of PBS buffer. Morphology of the exosomes was observed under a transmission electron microscope (H-7650, Hitachi, Tokyo, Japan). Concentration and size of the exosomes were measured by a nanoparticle tracking analyzer (Zetaview, Particle Metrux, Bavaria, Germany). The concentration of the exosomes was $1.66 \times 10^{8} / \mathrm{ml}$. The yield of exosomes was about $1.66 \times 10^{7}$ particles. The protein concentration was $1.63 \mu \mathrm{g} / \mu \mathrm{l}$, and the purity of exosomes was $1.02 \times 10^{5}$ particles/ $\mu$ g of protein. The exosomes were preserved at $-80^{\circ} \mathrm{C}$ for later use.

\section{DAH models induced by pristine}

The mice were randomized into the following four groups: the control group, DAH group, DAH+exo group and $\mathrm{DAH}+$ Methylprednisolone group. Each group was comprised of ten mice. Mice in the DAH group, DAH+ exo group and $\mathrm{DAH}+$ Methylprednisolone group were intraperitoneally injected with $0.5 \mathrm{ml}$ of sterile filtrated pristine (Sigma-Aldrich, Saint-Quentin Fallavier, France) for DAH model establishment. The pristine was purchased from Sigma-Aldrich (Saint-Quentin-Fallavier, France) and stored at room temperature. Mice in the control group were treated with $0.5 \mathrm{ml}$ of PBS. After DAH model establishment, mice in the DAH group and $\mathrm{DAH}+$ exo group were respectively injected with PBS (100 $\mu 1$ per mouse) and $0.2 \mathrm{mg} / \mathrm{ml} \mathrm{hUCMSC-exosome} \mathrm{suspension}(100 \mu \mathrm{l}$ per mouse) via tail vein every other day for 14 days. Mice in the $\mathrm{DAH}+$ Methylprednisolone group were intraperitoneally injected with $8 \mathrm{mg} / \mathrm{kg}$ methylprednisolone every other day; the concentration of methylprednisolone was reduced to $4 \mathrm{mg} / \mathrm{kg}$ after one week. Mice in the control group were left untreated. All the mice were euthanatized 14 days after DAH induction. Alveolar macrophages, lung tissue and bronchoalveolar lavage fluid (BALF) were extracted from the mice.

\section{Hematoxylin-eosin (H\&E) staining}

The paraffin block of mouse lung was cut into $5-\mu \mathrm{m}$ sections and dewaxed in xylene I $(15 \mathrm{~min})$ and xylene II (15 min). The dewaxed lung tissue was then washed in absolute alcohol for $2 \times 5 \mathrm{~min}$ and hydrated in gradient alcohol (from $95 \%, 80 \%$ to $70 \%$ ) for 5 min each time. After being washed, the tissue was stained in hematoxylin for $10 \mathrm{~min}$ and then washed in running water for $5 \mathrm{~min}$. Then the tissue was immersed in $1 \%$ hydrochloric acid-ethanol for $30 \mathrm{~s}$ and subjected to wash. After that, the tissue was stained in $1 \%$ eosin for 30 s. After being washed, the tissue was dehydrated in gradient alcohol (from 95\%, 95\%, 100\% to $100 \%$ ) for $1 \mathrm{~min}$ each time and permeabilized in xylene for $2 \times 3 \mathrm{~min}$. The tissue was mounted using neutral balsam and observed under a microscope.

\section{Enzyme-linked immunosorbent assay (ELISA)}

ELISA kits were purchased from Biolegend (San Diego, CA, USA) and applied to measure the expressions of IL-10, TGF- $\beta$, IL-6, TNF- $\alpha$ and IL- $\beta$ in BALF according to the manufacturer's instruction. Firstly, the kit was rewarmed to room temperature. The control $(50 \mu \mathrm{l})$ and BALF $(50 \mu \mathrm{l})$ were added to the plate for vibration at room temperature for $2 \mathrm{~h}$, after which the samples were subjected to the following steps: 4 washes, added with $100 \mu 1$ of diluted antibody detection solution, $1 \mathrm{~h}$ of vibration; 4 washes, added with $100 \mu 1$ of avdin-HRP solution, 30 min of vibration; 5 washes, added with $100 \mu 1$ of TMB substrate solution, $15 \mathrm{~min}$ of vibration. A microplate reader (Biotek Synergy2) was used to measure the absorbance at $450 \mathrm{~nm}$ within $30 \mathrm{~min}$ after the reaction was terminated. Quantities of those cytokines were counted according to the standard absorbance graph.

\section{Isolation of mouse alveolar macrophages}

The mouse was anesthetized using 10\% pentasorbital sodium. The trachea was exposed and incised in the middle. A type 9 needle was introduced into the trachea in the direction of the heart and fixed by fine-pointed tissue forceps. The lung was injected with $1 \mathrm{ml}$ of PBS via the trachea using a standard 1-ml syringe and gently massaged, after which the BALF was slowly pumped. This procedure was duplicated for five times. The BALF was centrifuged at $800 \mathrm{~g}$ for $10 \mathrm{~min}$. The cell pellet was resuspended in DMEM/F12 (gibco, USA) consisting of $10 \%$ FBS and $1 \%$ penicillin-streptomycin. Then the cells were cultured in an incubator at $37^{\circ} \mathrm{C}$ with $5 \% \mathrm{CO}_{2}$ and $95 \%$ humidity. Aliquots of the cell supernatant were preserved at $-20^{\circ} \mathrm{C}$.

\section{Test on phagocytosis of macrophage}

Carboxylate-modified red fluorescent latex beads (L3030, $2 \mu \mathrm{m}$, Sigma-Aldrich, St. Louis, MO USA) were diluted with $10 \%$ FBS-based DMEM to a concentration of $1: 100$ and then incubated at $37^{\circ} \mathrm{C}$ for $30 \mathrm{~min}$. Macrophages were mixed with the beads at a density of $1 \times 10^{5}$ cells $/ 100 \mu 1$ and incubated at $37^{\circ} \mathrm{C}$ for $4 \mathrm{~h}$. Then the macrophages were collected and washed twice in PBS. Fluorescence intensity of the macrophages was tested by flow cytometry. 


\section{Quantitative reverse transcription-polymerase chain reaction (qRT-PCR)}

The total RNA was extracted from macrophages using TRIZOL reagent (Life Technologies, NY, USA) and the RNA concentration and purity were measured by a microplate reader (Biotek Synergy2). A PCR amplification instrument was used to synthesize cDNA. The real-time qRT-PCR was performed using a fluorescent quantitative PCR analyzer (CFX Connect, Bio-Rad, USA). GAPDH served as the internal control of mRNA. The reaction conditions were as follows: predegeneration $(10 \mathrm{~min})$ at $95^{\circ} \mathrm{C}$; 40 cycles of degeneration $(10 \mathrm{~s})$ at $95^{\circ} \mathrm{C}$, annealing $(20 \mathrm{~s})$ at $60^{\circ} \mathrm{C}$ and extension $(34 \mathrm{~s})$ at $72^{\circ} \mathrm{C}$. The statistics were analyzed using $2^{-\Delta \Delta \mathrm{Ct}}$ method according to the following formula: $\Delta \Delta \mathrm{Ct}=[\mathrm{Ct}(\text { target gene })-\mathrm{Ct}(\text { reference gene })]_{\text {experimental group }}{ }^{-}$ $[\mathrm{Ct}(\text { target gene })-\mathrm{Ct}(\text { reference gene })]_{\text {control group. Primers }}$ used in the PCR were synthesized by Sangon Biotech (Shanghai, China) and the primer sequences are shown in Table 1.

\section{Western blot}

Concentration of the proteins extracted from the isolated exosomes was measured using a BCA kit (Vazyme Biotech Co., Ltd., Nanjing, China). The proteins were separated by electrophoresis in 10\% SDS-polyacrylamide gel and then transferred onto a polyvinylidene fluoride (PVDF) membrane (Millipore, Billerica, MA). The proteins were confined in 5\% skim milk at room temperature for $1 \mathrm{~h}$. After that, the membrane was added with rat anti human

Table 1. Primer sequences

\begin{tabular}{ll}
\hline Name of primer & \multicolumn{1}{c}{ Sequences } \\
\hline iNOS-F & 5' CCCTTCAATGGTTGGTACATGG 3' \\
iNOS-R & 5' CATTGATCTCCGTGACAGCC 3' \\
IL-6-F & 5' CACCACTGCCCGTAGATGAAG 3' \\
IL-6-R & 5' AGGGTACAGTCGTCGAACTCT 3' \\
TNF- $\alpha-\mathrm{F}$ & 5' TGCTTGTTCCTCAGCCTCTT 3' \\
TNF- $\alpha-\mathrm{R}$ & 5' CAGAGGGCTGATTAGAGAGAGGT $3^{\prime}$ \\
IL-1 $\beta-\mathrm{F}$ & 5' TGAGCCAAGACAGAAAACCCA 3' \\
IL-1 $\beta-\mathrm{R}$ & 5' GGGACATGAGTAAGGTTCCTGT 3' \\
Arg1-F & 5' CATGGGCAACCTGTGTCCTT 3' \\
Arg1-R & 5' CGATGTCTTTGGCAGATATGCA 3' \\
IL-10-F & 5' GAGATGCCTTCAGCAGAGTGAAGA 3' \\
IL-10-R & 5' AGTTCACATGCGCCTTGATGTC 3' \\
TGF- $\beta-F$ & 5' GCGACTCGCCAGAGTGGTTA 3' \\
TGF- $\beta-\mathrm{R}$ & 5' GTTGATGTCCACTTGCAGTGTGTTA 3' \\
chi3I3-F & 5' TGGCCCACCAGGAAAGTACA 3' \\
chi3I3-R & 5' CAGTGGCTCCTTCATTCAGAAA 3' \\
GAPDH-F & 5' GTCGATGGCTAGTCGTAGCATCGAT 3' \\
GAPDH-R & 5' TGCTAGCTGGCATGCCCGATCGATC3' \\
\hline
\end{tabular}

F: forward, R: reverse. antibodies of ALIX (ab117600, $1: 1,000)$, CD63 (ab59479, $1: 1,000)$ and TSG101 (ab83, $1: 1,000)$ (Abcam, Cambridge, MA, USA) and incubated at $4^{\circ} \mathrm{C}$ overnight. After $3 \times 10$ min TBST wash, the proteins were incubated with horse radish peroxidase labeled goat anti rat IgG antibody (1 : 5,000, ComWin Biotech Co., Ltd., Beijing, China) at room temperature for $1 \mathrm{~h}$ and subjected to $3 \times 10 \mathrm{~min}$ TBST wash. The protein expressions were analyzed by a chemiluminescence imaging system (Tanon Science \& Technology Co., Ltd., Shanghai, China).

\section{Flow cytometry}

Single cell suspension of primary mouse alveolar macrophages $\left(1 \times 10^{6}\right.$ cells $\left./ 100 \mu \mathrm{l}\right)$ was fixed in $4 \%$ paraformaldehyde for $15 \mathrm{~min}$. After that, the macrophage suspension was incubated with rabbit anti CD1lb-FITC antibody (M1/70, $10 \mu 1$, BD Bioscience, USA), monoclonal F4/80-PerCP-Cyanine5.5 antibody (BM8, $10 \mu 1)$, monoclonal CD86-PE antibody (B7-2, $10 \mu 1)$ and polyclonal CD206-APC antibody (PA5-46879, $10 \mu \mathrm{l}$ ) (eBioscience, USA) at $4^{\circ} \mathrm{C}$ in the dark for $10 \mathrm{~min}$. The proportions of $\mathrm{F} 4 / 80^{+} \mathrm{CD} 11 \mathrm{~b}^{+} \mathrm{CD} 86^{+} \mathrm{CD} 206^{-}$cells (M1 macrophages) and $\mathrm{F} 4 / 80^{+} \mathrm{CD} 11 \mathrm{~b}^{+} \mathrm{CD} 86^{-} \mathrm{CD} 206^{+}$cells (M2 macrophages) were measured by the FACS Calibur ${ }^{\mathrm{TM}}$ system (BD, USA).

\section{Statistical analysis}

All records were statistically analyzed on SPSS 17.0 and presented in mean \pm SD (standard deviation). Group differences were analyzed using One-way analysis of variance (ANOVA). The LSD method was applied for homogeneity test of variances and the Games-Howell method was for heterogeneity test of variances. $\mathrm{p}<0.05$ was considered statistically significant.

\section{Results}

\section{Identification of hUCMSC-exosomes}

Particles isolated from hUCMSCs were in round or elliptical shape with an intact capsule (Fig. 1A). These particles had positive expression of exosome makers Alix, CD63 and TSG101 (Fig. 1B). The particle diameter ranged from $40 \sim 120 \mathrm{~nm}$ with an average value of $100 \mathrm{~nm}$; the average and maximum diameters were consistent with those of exosomes (Fig. 1C). From the above, these isolated particles were hUCMSC-exosomes.

\section{Evaluation of DAH models}

H\&E staining showed that there were slight hemorrhage and neutrophilic vasculitis in the mouse lung after pris- 
tine treatment (Fig. 2A) Pro-inflammatory cytokines IL-6 and TNF- $\alpha$ were increased and anti-inflammatory cytokines IL-10 and TGF- $\beta$ were decreased in the BALF of $\mathrm{DAH}$ mice compared to the control group (Fig. $2 \mathrm{~B} \sim \mathrm{E}$, $\mathrm{p}<0.01)$. The above results indicate successful establishment of DAH models.

A

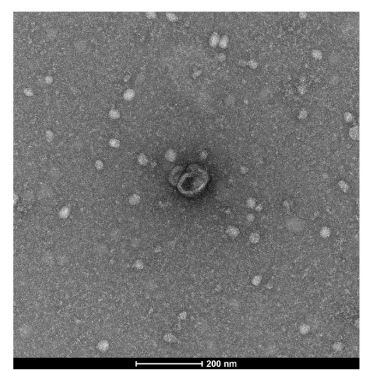

C

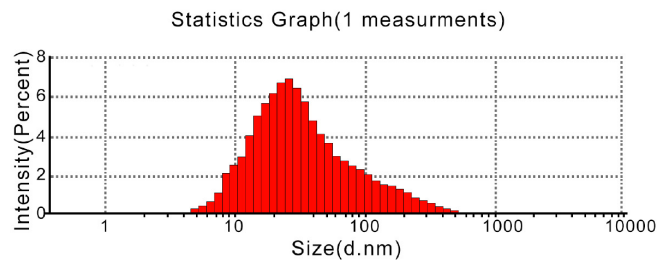

Fig. 1. Identification of hUCMSC-exosomes. (A) The ultrastructure of exosomes was observed under a transmission electron microscope; (B) expressions of exosome markers were tested by Western blotting; (C) exosome size was measured by the nanoparticle tracking analyzer Zetaview. hUCMSC, human umbilical cord mesenchymal stem cell.

\section{hUCMSC-exosomes alleviate pathological symptoms of DAH mice}

The hemorrhage and small-vessel vasculitis in mouse lung were attenuated 14 days after hUCMSC-exosome injection or methylprednisolone treatment (Fig. 3A). ELISA showed that IL- 6 and TNF- $\alpha$ were decreased while IL-10 and TGF- $\beta$ were induced in the DAH group compared to the control group $(\mathrm{p}<0.01)$; the anti-inflammatory effect of methylprednisolone was slightly stronger than that of hUCMSC-exosomes, but the difference was insignificant (Fig. 3B E). hUCMSC-exosomes can lighten the pathological symptoms of DAH mice.

\section{hUCMSC-exosomes enhance M2 polarization to attenuate DAH}

Alveolar macrophages were isolated from the mice. Expression of macrophage markers and phenotype of macrophage were detected to investigate the mechanism underlying the effect of hUCMSC-exosomes on DAH. Macrophages in the DAH group highly expressed iNOS, IL-6, TNF- $\alpha$ and IL-1 $\beta$ compared to the control group (Fig. 4A $\sim \mathrm{D}, \mathrm{p}<0.01$ ), exhibiting $\mathrm{Ml}$ macrophage phenotype. After hUCMSC-exosome or methylprednisolone treatment, iNOS, IL-6, TNF- $\alpha$ and IL-1 $\beta$ were decreased in macrophages (Fig. 4A $\sim \mathrm{D}, \mathrm{p}<0.01$ ) while Arg1, IL-10, TGF- $\beta$ and chi313 were increased (Fig. $4 \mathrm{E} \sim \mathrm{H}, \mathrm{p}<0.01$ ), presenting M2 macrophage phenotype.

Flow cytometry was applied to test the phenotypic transformation of primary mouse alveolar macrophages

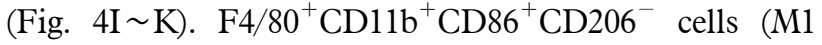
macrophages) were increased in the DAH group compared to the control group $(31.0 \pm 2.0 \%$ vs. $0.52 \pm 0.34 \%, \mathrm{p}<0.01)$.
A

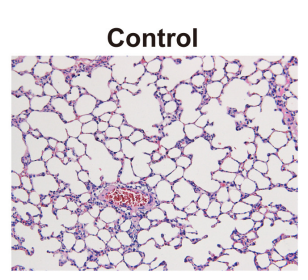

C



D

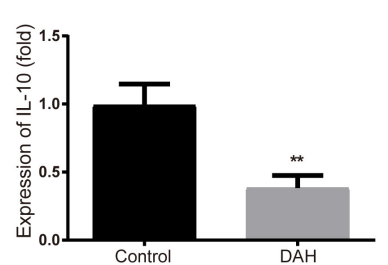

Control

B

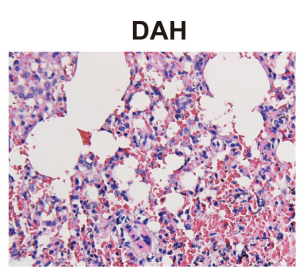

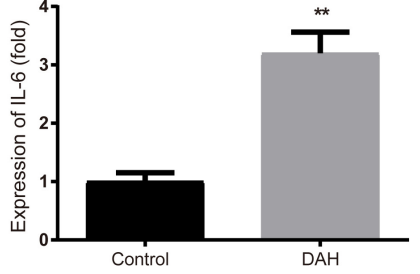

E



Fig. 2. Establishment of DAH models on mice. (A) H\&E staining detected the pathological conditions of mouse lung; $(B \sim E)$ ELISA measured the expressions of IL-6, TNF- $\alpha$, IL-10 and TGF- $\beta$ in mouse BALF. Each group had ten mice. ${ }^{* *} \mathrm{p}<$ 0.01 compared to the control group; $\mathrm{DAH}$, diffuse alveolar hemorrhage; BALF, bronchoalveolar lavage fluid; ELISA, enzyme-linked immunosorbent assay. 
A

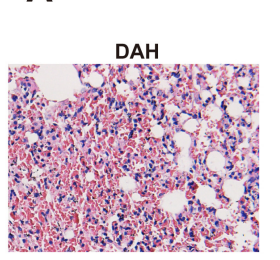

C

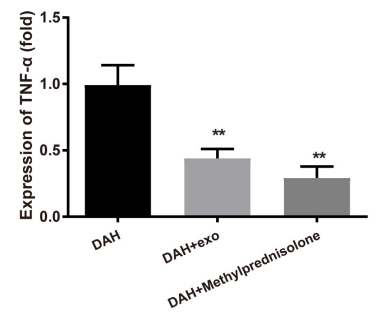

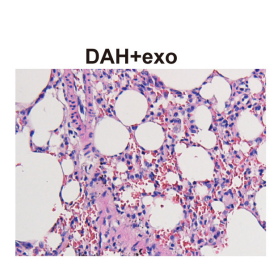

D

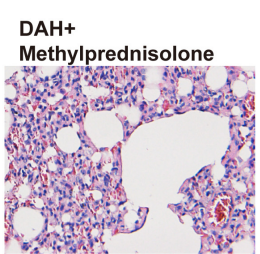

E

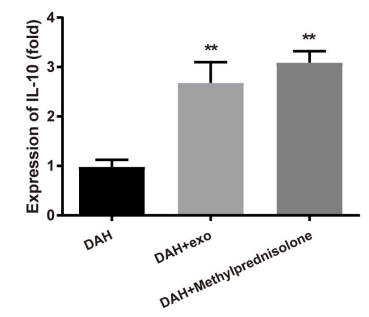

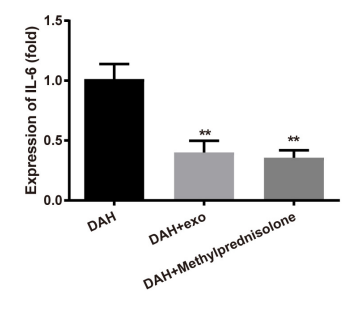

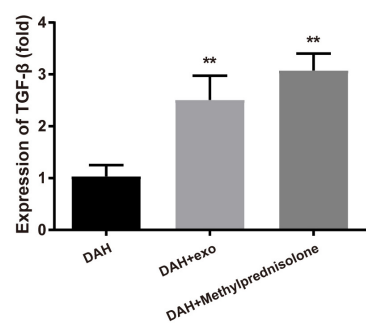

Fig. 3. hUCMSC-exosomes alleviate pathological symptoms of DAH mice. After hUCMSC-exosome or methylprednisolone treatment, (A) $\mathrm{H} \& \mathrm{E}$ staining assessed the morphology of mouse lung; $(B \sim E)$ ELISA measured the expressions of IL-6, TNF- $\alpha$, IL-10 and TGF- $\beta$ in mouse BALF. Each group had ten mice. ${ }^{* *} \mathrm{p}<0.01$ compared to the control group; hUCMSC, human umbilical cord mesenchymal stem cell; DAH, diffuse alveolar hemorrhage; BALF, bronchoalveolar lavage fluid; ELISA, enzyme-linked immunosorbent assay.
After hUCMSC-exosome treatment, $\mathrm{F} 4 / 80^{+} \mathrm{CD} 11 \mathrm{~b}^{+} \mathrm{CD} 86$ ${ }^{+} \mathrm{CD}_{206}{ }^{-}$cells were reduced $(2.81 \pm 0.67 \%$ vs. $31.0 \pm 2.0 \%)$ while $\mathrm{F} 4 / 80^{+} \mathrm{CD} 11 \mathrm{~b}^{+} \mathrm{CD} 86^{-} \mathrm{CD} 206^{+}$cells (M2 macrophages) were increased $(21.0 \pm 3.0 \%$ vs. $5.67 \pm 1.53 \%, \mathrm{p}<0.01)$, suggesting that $\mathrm{M} 2$ polarization was associated with the regulatory effect of hUCMSC-exosomes in DAH.

The macrophages were incubated with fluorescent beads to evaluate their phagocytosis capacity. The phagocytosis was impeded by DAH compared to the control group while enhanced in the $\mathrm{DAH}+$ exo group and $\mathrm{DAH}+$ Methylprednisolone group compared to the DAH group (Fig. 4L). Both hUCMSC-exosomes and methylprednisolone can improve the phagocytosis of macrophages in DAH. Taken together, hUCMSC-exosomes facilitated the transformation of macrophages from M1 to M2 phenotype and further enhanced the macrophage phagocytosis.

\section{Discussion}

$\mathrm{DAH}$ is a life-threatening pulmonary complication which usually occurs at the active stage of SLE (31). DAH is mostly associated with pulmonary capillaritis which is confined to the pulmonary microcirculation (32). Currently, no specific therapy approach for SLE-DAH has been approved by the Food and Drug Administration (33). Therefore, ongoing studies into the management of SLEDAH are needed to improve the clinic outcomes of SLEDAH. This research decodes the mechanism underlying the suppressive effect of hUCMSC-exosomes on DAH.

Pristane was applied in our research to establish DAH models in C57BL/6J mice. Pristane is an isoprenoid al- kane produced in plants, shark liver and mineral oil (34). Intraperitoneal injection of pristine stimulates chronic inflammation in mice thereby causing a lupus-like disease (35). Pristine can induce pulmonary hemorrhage in C57BL/6 mice along with infiltration of inflammatory cells (36). In the present study, H\&E staining detected that there were slight hemorrhage and small-vessel vasculitis characterized by assembly of neutrophils in pristine-induced DAH. Furthermore, pro-inflammatory cytokines (IL-6 and TNF- $\alpha$ ) were increased while anti-inflammatory cytokines (IL-10 and TGF- $\beta$ ) were decreased in BALF of DAH mice, suggesting pristine treatment triggered inflammation response in the mice.

After injection of hUCMSC-exosomes, the hemorrhage and small-vessel vasculitis in $\mathrm{DAH}$ mice were reduced, and in the meantime pro-inflammatory cytokines were decreased and anti-inflammatory cytokines were increased in the BALF. MSCs have shown great potential for anti-inflammation and immune-modulation in massive clinical trials (37). Increasing evidence has demonstrated that MSC-derived exosomes have similar therapeutic functions to those of MSCs (38). Shi et al. (39) found that UCMSC transplantation improved the conditions of patients with SLE-DAH, which was manifested by amelioration of hematological status, oxygen saturation and alveolar infiltrates. EVs secreted by MSCs have been documented to prevent acute lung injury as well as inflammatory lung diseases (40). MSC-derived exosomes can also suppress inflammatory responses in many other disease conditions such as myocardial infarction (41), burn-induced inflammation (42) and spinal cord injury (43). 

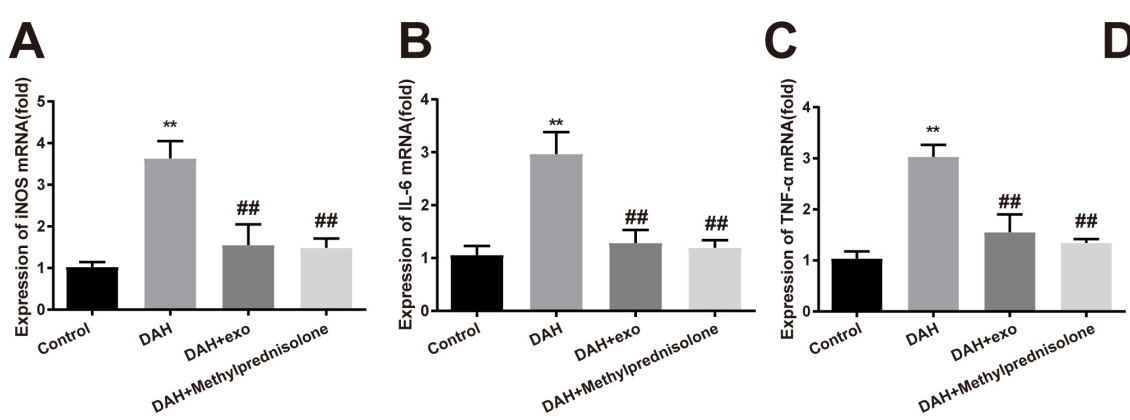

D

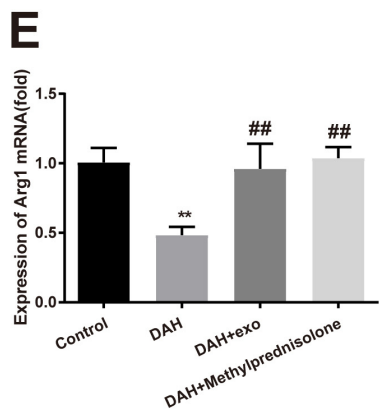

F

G

H


I
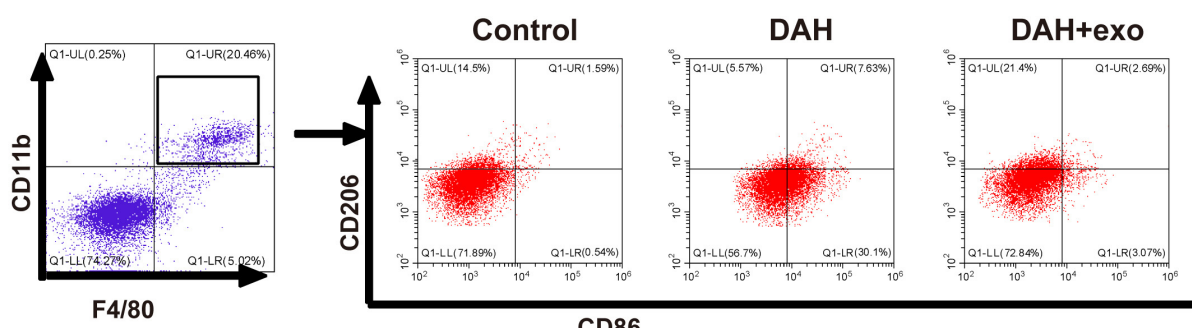

DAH+
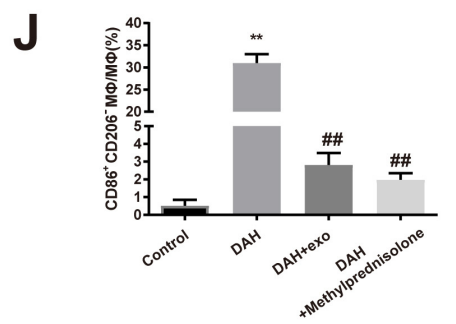

K

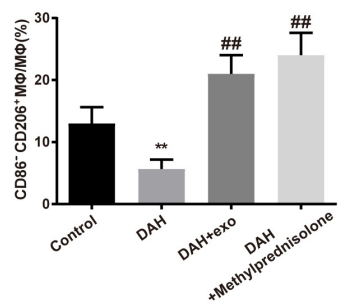

L

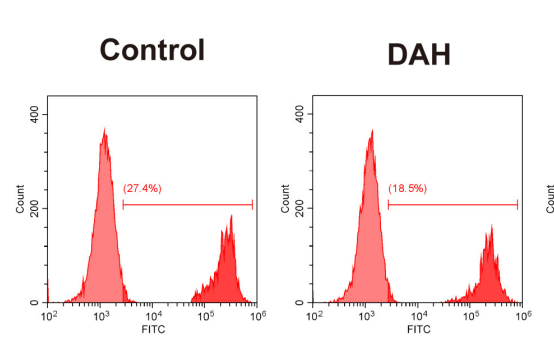

\section{DAH+exo}

\section{DAH+}
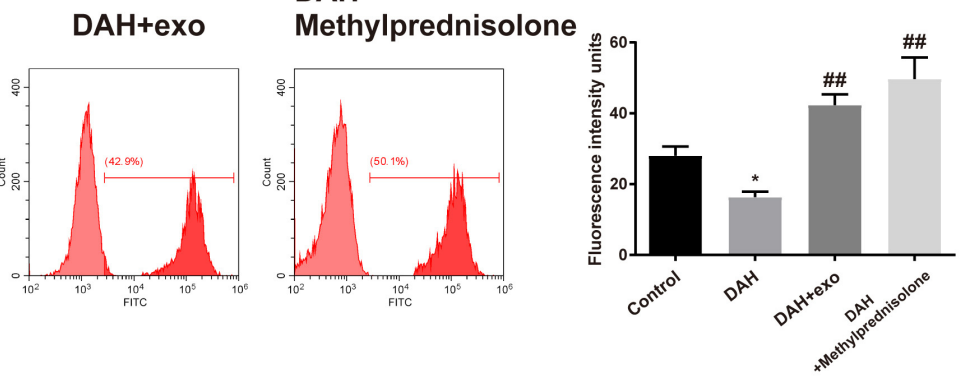

Fig. 4. hUCMSC-exosomes facilitate the transformation of macrophages from $M 1$ into $M 2$ phenotype. (A $\sim H)$ The expressions of iNOS, IL-6, TNF- $\alpha$, IL-1 $\beta, \operatorname{Arg} 1$, IL-10, TGF- $\beta$ and chi3l3 in mouse macrophages were tested by qRT-PCR; (I $\sim \mathrm{K})$ the proportions of M1 and M2 macrophages were measured by flow cytometry; (L) phagocytosis was assessed by flow cytometry. $\mathrm{n}=10 ;{ }^{*} \mathrm{p}<0.05,{ }^{* *} \mathrm{p}<0.01$ compared to the control group; $\# \mathrm{p}<0.05, \# \# \mathrm{p}<0.01$ compared to the DAH group; hUCMSC, human umbilical cord mesenchymal stem cell; $\mathrm{DAH}$, diffuse alveolar hemorrhage. 
As macrophages are implicated in the regulation of inflammation, we supposed that hUCMSC-exosomes acted on DAH by activating specific macrophage phenotype. Macrophages are usually sorted into classically-activated (M1) macrophages and alternatively-activated (M2) macrophages. M1 macrophages are characterized by high expressions of CD80, CD86, COX-2 and iNOS and they produce pro-inflammatory cytokines such as TNF- $\alpha$, IL1- $\beta$ and IL-6, thus starting and sustaining inflammatory responses; on the other hand, M2 macrophages express high level of CD206 and release TGF- $\beta$ to suppress inflammation and promote wound repair (44). Based on the above evidence, alveolar macrophages in DAH mice presented M1 phenotype, which was manifested by high levels of iNOS, IL-6, TNF- $\alpha$ and IL-1 $\beta$. After hUCMSCexosome treatment, these pro-inflammatory factors were reduced and M2 macrophages were activated. Flow cytometry also measured out an increase in the proportion of M2 macrophages post hUCMSC-exosome injection, suggesting M1 macrophage polarized to M2 macrophage. In addition, the phagocytosis of macrophages was enhanced in DAH mice treated with hUCMSC-exosomes.

Previous studies have verified that macrophage polarization plays a vital role in different medical conditions. Liver X receptor antagonist prevented DAH in murine lupus by activating M2 macrophage polarization (45). MSC-derived exosomes ameliorated hyperoxia-induced lung injuries and inflammation through immunomodulation of macrophage phenotype (46). Exosomes derived from LPS-preconditioned MSCs relieved inflammation and facilitated wound healing in a M2 macrophage-dependent manner by shuttling let-7b (47). MSC-exosomes also drive the macrophage polarization in many other diseases such as breast cancer (48), myocardial infarction (49) and atherosclerosis (50). Nonetheless, there has been no report of the mediation of MSC-exosomes on macrophage polarization in SLE-DAH. The current study is the first to claim that the macrophage polarization can be regulated by MSC-exosomes in SLE-DAH.

In summary, hUCMSC-exosomes attenuate symptoms of SLE-DAH by preventing pulmonary inflammation via macrophage polarization. hUCMSC-exosomes activate M2 macrophage to suppress inflammatory responses in the BALF. It is worth mentioning that hUCMSC-exosomes have a similar effect to that of the anti-inflammatory agent methylprednisolone. Understanding of the underlying mechanism of hUCMSC-exosomes may provide new therapeutic targets for treatment of DAH and broaden the prospects of this exosome-based cell-free therapy.

\section{Acknowledgments}

Thanks for all the contributors and participants.

This research was supported by the grants from the Project to improve the basic Scientific Research ability of Young and Middle-aged Teachers in Colleges and Universities in Guangxi in 2020 (2020KY03025) and Innovation Project of Guangxi Graduate Education (YCBZ2020047).

\section{Potential Conflict of Interest}

The authors have no conflicting financial interest.

\section{References}

1. Cucuzza ME, Marino SD, Schiavone L, Smilari P, Filosco F, Barone P. Diffuse alveolar haemorrage as initial presentation of systemic lupus erythematosus: a case report. Lupus 2018;27:507-510

2. Al-Adhoubi NK, Bystrom J. Systemic lupus erythematosus and diffuse alveolar hemorrhage, etiology and novel treatment strategies. Lupus 2020;29:355-363

3. Kim D, Choi J, Cho SK, Choi CB, Kim TH, Jun JB, Yoo $\mathrm{DH}$, Bae SC, Sung YK. Clinical characteristics and outcomes of diffuse alveolar hemorrhage in patients with systemic lupus erythematosus. Semin Arthritis Rheum 2017; 46:782-787

4. Ednalino C, Yip J, Carsons SE. Systematic review of diffuse alveolar hemorrhage in systemic lupus erythematosus: focus on outcome and therapy. J Clin Rheumatol 2015;21: 305-310

5. Parmar M. Towards stem cell based therapies for Parkinson's disease. Development 2018;145:dev156117

6. Senior PA, Pettus JH. Stem cell therapies for Type 1 diabetes: current status and proposed road map to guide successful clinical trials. Diabet Med 2019;36:297-307

7. Jin MC, Medress ZA, Azad TD, Doulames VM, Veeravagu A. Stem cell therapies for acute spinal cord injury in humans: a review. Neurosurg Focus 2019;46:E10

8. Samsonraj RM, Raghunath M, Nurcombe V, Hui JH, van Wijnen AJ, Cool SM. Concise review: multifaceted characterization of human mesenchymal stem cells for use in regenerative medicine. Stem Cells Transl Med 2017;6:21732185

9. Zou Q, Wu M, Zhong L, Fan Z, Zhang B, Chen Q, Ma F. Development of a xeno-free feeder-layer system from human umbilical cord mesenchymal stem cells for prolonged expansion of human induced pluripotent stem cells in culture. PLoS One 2016;11:e0149023

10. Can A, Celikkan FT, Cinar O. Umbilical cord mesenchymal stromal cell transplantations: a systemic analysis of clinical trials. Cytotherapy 2017;19:1351-1382

11. Liang J, Gu F, Wang H, Hua B, Hou Y, Shi S, Lu L, Sun L. Mesenchymal stem cell transplantation for diffuse alveolar hemorrhage in SLE. Nat Rev Rheumatol 2010;6:486-489

12. Hessvik NP, Llorente A. Current knowledge on exosome 
biogenesis and release. Cell Mol Life Sci 2018;75:193-208

13. Gurunathan S, Kang MH, Jeyaraj M, Qasim M, Kim JH. Review of the isolation, characterization, biological function, and multifarious therapeutic approaches of exosomes. Cells 2019;8:307

14. Li P, Kaslan M, Lee SH, Yao J, Gao Z. Progress in exosome isolation techniques. Theranostics 2017;7:789-804

15. Zhang R, Zhu Y, Li Y, Liu W, Yin L, Yin S, Ji C, Hu Y, Wang Q, Zhou X, Chen J, Xu W, Qian H. Human umbilical cord mesenchymal stem cell exosomes alleviate sepsis-associated acute kidney injury via regulating microRNA146b expression. Biotechnol Lett 2020;42:669-679

16. Zhang X, Liu J, Yu B, Ma F, Ren X, Li X. Effects of mesenchymal stem cells and their exosomes on the healing of large and refractory macular holes. Graefes Arch Clin Exp Ophthalmol 2018;256:2041-2052

17. Wu Y, Qiu W, Xu X, Kang J, Wang J, Wen Y, Tang X, Yan Y, Qian H, Zhang X, Xu W, Mao F. Exosomes derived from human umbilical cord mesenchymal stem cells alleviate inflammatory bowel disease in mice through ubiquitination. Am J Transl Res 2018;10:2026-2036

18. Ding $M$, Shen Y, Wang P, Xie Z, Xu S, Zhu Z, Wang Y, Lyu Y, Wang D, Xu L, Bi J, Yang H. Exosomes isolated from human umbilical cord mesenchymal stem cells alleviate neuroinflammation and reduce amyloid-beta deposition by modulating microglial activation in Alzheimer's disease. Neurochem Res 2018;43:2165-2177

19. Yaghoubi Y, Movassaghpour A, Zamani M, Talebi M, Mehdizadeh A, Yousefi M. Human umbilical cord mesenchymal stem cells derived-exosomes in diseases treatment. Life Sci 2019;233:116733

20. Sun G, Li G, Li D, Huang W, Zhang R, Zhang H, Duan $\mathrm{Y}$, Wang B. hucMSC derived exosomes promote functional recovery in spinal cord injury mice via attenuating inflammation. Mater Sci Eng C Mater Biol Appl 2018;89:194204

21. Fang S, Xu C, Zhang Y, Xue C, Yang C, Bi H, Qian X, Wu M, Ji K, Zhao Y, Wang Y, Liu H, Xing X. Umbilical cord-derived mesenchymal stem cell-derived exosomal microRNAs suppress myofibroblast differentiation by inhibiting the transforming growth factor- $\beta / \mathrm{SMAD} 2$ pathway during wound healing. Stem Cells Transl Med 2016;5: $1425-1439$

22. Perez-Hernandez J, Redon J, Cortes R. Extracellular vesicles as therapeutic agents in systemic lupus erythematosus. Int J Mol Sci 2017;18:717

23. Zhou D, Huang C, Lin Z, Zhan S, Kong L, Fang C, Li J. Macrophage polarization and function with emphasis on the evolving roles of coordinated regulation of cellular signaling pathways. Cell Signal 2014;26:192-197

24. Shapouri-Moghaddam A, Mohammadian S, Vazini H, Taghadosi M, Esmaeili SA, Mardani F, Seifi B, Mohammadi A, Afshari JT, Sahebkar A. Macrophage plasticity, polarization, and function in health and disease. J Cell Physiol 2018;233:6425-6440

25. Wang N, Liang H, Zen K. Molecular mechanisms that in- fluence the macrophage m1-m2 polarization balance. Front Immunol 2014;5:614

26. Barros MH, Hauck F, Dreyer JH, Kempkes B, Niedobitek G. Macrophage polarisation: an immunohistochemical approach for identifying $\mathrm{M} 1$ and $\mathrm{M} 2$ macrophages. PLoS One 2013;8:e80908

27. Xu R, Zhang F, Chai R, Zhou W, Hu M, Liu B, Chen X, Liu $\mathrm{M}, \mathrm{Xu} \mathrm{Q}$ Liu N, Liu S. Exosomes derived from pro-inflammatory bone marrow-derived mesenchymal stem cells reduce inflammation and myocardial injury via mediating macrophage polarization. J Cell Mol Med 2019;23:76177631

28. Zhao J, Li X, Hu J, Chen F, Qiao S, Sun X, Gao L, Xie J, Xu B. Mesenchymal stromal cell-derived exosomes attenuate myocardial ischaemia-reperfusion injury through miR-182-regulated macrophage polarization. Cardiovasc Res 2019;115:1205-1216

29. Liu H, Liang Z, Wang F, Zhou C, Zheng X, Hu T, He $\mathrm{X}$, Wu X, Lan P. Exosomes from mesenchymal stromal cells reduce murine colonic inflammation via a macrophage-dependent mechanism. JCI Insight 2019;4:e131273

30. Zhuang H, Han S, Lee PY, Khaybullin R, Shumyak S, Lu L, Chatha A, Afaneh A, Zhang Y, Xie C, Nacionales D, Moldawer L, Qi X, Yang LJ, Reeves WH. Pathogenesis of diffuse alveolar hemorrhage in murine lupus. Arthritis Rheumatol 2017;69:1280-1293

31. Shen M, Zeng X, Tian X, Zhang F, Zeng X, Zhang X, Xu W. Diffuse alveolar hemorrhage in systemic lupus erythematosus: a retrospective study in China. Lupus 2010;19: 1326-1330

32. Park MS. Diffuse alveolar hemorrhage. Tuberc Respir Dis (Seoul) 2013;74:151-162

33. Morales-Nebreda L, Alakija O, Ferguson KT, Singer BD. Systemic lupus erythematosus-associated diffuse alveolar hemorrhage: a case report and review of the literature. Clin Pulm Med 2018;25:166-169

34. Zhuang H, Szeto C, Han S, Yang L, Reeves WH. Animal models of interferon signature positive lupus. Front Immunol 2015;6:291

35. Reeves WH, Lee PY, Weinstein JS, Satoh M, Lu L. Induction of autoimmunity by pristane and other naturally occurring hydrocarbons. Trends Immunol 2009;30:455-464

36. Barker TT, Lee PY, Kelly-Scumpia KM, Weinstein JS, Nacionales DC, Kumagai Y, Akira S, Croker BP, Sobel ES, Reeves WH, Satoh M. Pathogenic role of B cells in the development of diffuse alveolar hemorrhage induced by pristane. Lab Invest 2011;91:1540-1550

37. Pittenger MF, Discher DE, Péault BM, Phinney DG, Hare JM, Caplan AI. Mesenchymal stem cell perspective: cell biology to clinical progress. NPJ Regen Med 2019;4:22

38. Pashoutan Sarvar D, Shamsasenjan K, Akbarzadehlaleh P. Mesenchymal stem cell-derived exosomes: new opportunity in cell-free therapy. Adv Pharm Bull 2016;6:293-299

39. Shi D, Wang D, Li X, Zhang H, Che N, Lu Z, Sun L. Allogeneic transplantation of umbilical cord-derived mesenchymal stem cells for diffuse alveolar hemorrhage in sys- 
temic lupus erythematosus. Clin Rheumatol 2012;31:841846

40. Monsel A, Zhu YG, Gudapati V, Lim H, Lee JW. Mesenchymal stem cell derived secretome and extracellular vesicles for acute lung injury and other inflammatory lung diseases. Expert Opin Biol Ther 2016;16:859-871

41. Teng X, Chen L, Chen W, Yang J, Yang Z, Shen Z. Mesenchymal stem cell-derived exosomes improve the microenvironment of infarcted myocardium contributing to angiogenesis and anti-inflammation. Cell Physiol Biochem 2015;37:2415-2424

42. Li X, Liu L, Yang J, Yu Y, Chai J, Wang L, Ma L, Yin H. Exosome derived from human umbilical cord mesenchymal stem cell mediates miR-181c attenuating burn-induced excessive inflammation. EBioMedicine 2016;8:72-82

43. Huang JH, Yin XM, Xu Y, Xu CC, Lin X, Ye FB, Cao $\mathrm{Y}$, Lin FY. Systemic administration of exosomes released from mesenchymal stromal cells attenuates apoptosis, inflammation, and promotes angiogenesis after spinal cord injury in rats. J Neurotrauma 2017;34:3388-3396

44. Viola A, Munari F, Sánchez-Rodríguez R, Scolaro T, Castegna A. The metabolic signature of macrophage responses. Front Immunol 2019;10:1462

45. Han S, Zhuang H, Shumyak S, Wu J, Xie C, Li H, Yang LJ, Reeves WH. Liver X receptor agonist therapy prevents diffuse alveolar hemorrhage in murine lupus by repolarizing macrophages. Front Immunol 2018;9:135

46. Willis GR, Fernandez-Gonzalez A, Anastas J, Vitali SH,
Liu X, Ericsson M, Kwong A, Mitsialis SA, Kourembanas S. Mesenchymal stromal cell exosomes ameliorate experimental bronchopulmonary dysplasia and restore lung function through macrophage immunomodulation. Am J Respir Crit Care Med 2018;197:104-116

47. Ti D, Hao H, Tong C, Liu J, Dong L, Zheng J, Zhao Y, Liu H, Fu X, Han W. LPS-preconditioned mesenchymal stromal cells modify macrophage polarization for resolution of chronic inflammation via exosome-shuttled let-7b. J Transl Med 2015;13:308

48. Biswas S, Mandal G, Roy Chowdhury S, Purohit S, Payne KK, Anadon C, Gupta A, Swanson P, Yu X, Conejo-Garcia JR, Bhattacharyya A. Exosomes produced by mesenchymal stem cells drive differentiation of myeloid cells into immunosuppressive $\mathrm{m} 2$-polarized macrophages in breast cancer. J Immunol 2019;203:3447-3460

49. Deng S, Zhou X, Ge Z, Song Y, Wang H, Liu X, Zhang D. Exosomes from adipose-derived mesenchymal stem cells ameliorate cardiac damage after myocardial infarction by activating S1P/SK1/S1PR1 signaling and promoting macrophage M2 polarization. Int J Biochem Cell Biol 2019;114: 105564

50. Li J, Xue H, Li T, Chu X, Xin D, Xiong Y, Qiu W, Gao X, Qian M, Xu J, Wang Z, Li G. Exosomes derived from mesenchymal stem cells attenuate the progression of atherosclerosis in $\mathrm{ApoE}^{-} /$mice via miR-let7 mediated infiltration and polarization of M2 macrophage. Biochem Biophys Res Commun 2019;510:565-572 